\title{
Reconstrucción de la vegetación posglacial y su relación con el ascenso relativo del nivel del mar en el extremo este del canal Beagle, Tierra del Fuego, Argentina
}

\author{
Ana María Borromei ${ }^{1}$, Juan Federico Ponce ${ }^{2}$, Andrea Coronato ${ }^{2,3}$, María Soledad Candel ${ }^{1}$, \\ Daniela Olivera ${ }^{4}$, Masaaki Okuda ${ }^{5}$
}

\author{
Instituto de Geología del Sur (INGEOSUR-CONICET), Departamento de Geología, Universidad Nacional del Sur, San Juan 670, \\ B8000ICN Bahía Blanca, Argentina. \\ borromei@criba.edu.ar; scandel@uns.edu.ar \\ 2 Centro Austral de Investigaciones Cientificas (CADIC-CONICET), B. Houssay 200, 9410, Ushuaia, Tierra del Fuego, Argentina. \\ jfedeponce@cadic-conicet.gob.ar; acoronato@cadic-conicet.gob.ar \\ 3 Instituto de Desarrollo Económico e Innovación, Universidad Nacional de Tierra del Fuego, Onas 450, 9410 Ushuaia, Argentina. \\ ${ }^{4}$ Laboratorio de Palinología, Departamento de Geología, Universidad Nacional del Sur, San Juan 670, B8000ICN Bahía Blanca, Argentina. \\ danielaolivera2000@yahoo.com.ar \\ ${ }_{5}$ Natural History Museum and Institute, Chiba, 955-2 Aoba-cho, Chuo-ku, Chiba 260-8682, Japan. \\ okuda@chiba-muse.or.jp
}

*Corresponding author: borromei@criba.edu.ar

\begin{abstract}
RESUMEN. A partir del análisis polínico de una turbera de Donatia-Astelia ubicada en Punta Moat (Moat-2; 54 ${ }^{\circ} 58^{\text {' }}$ 50"S, 66 $44^{\prime} 10^{\prime \prime} \mathrm{W} ; 40 \mathrm{~m}$ s.n.m.), este del canal Beagle, y de modelos digitales de terreno vinculados a la curva de ascenso del mar posglacial, se reconstruye la evolución de la línea de costa y la vegetación desde el Último Máximo Glacial (UMG, ca. $25 \mathrm{ka}$ cal AP) hasta el Holoceno medio (5.000 años cal AP). La evolución de las comunidades vegetales en el extremo este del canal Beagle coincide en líneas generales con el modelo regional de instalación posglacial de la vegetación en las áreas desprovistas de hielo del sur de Tierra del Fuego. Este modelo plantea la sucesión de comunidades pioneras graminosas y arbustivas propias de la estepa, el ecotono bosque-estepa y el bosque de Nothofagus. Con anterioridad a los 17.700 años cal AP, el inicio de la deglaciación en Punta Moat y el retiro de los paleoglaciares alpinos hacia las cabeceras de las sierras de Lucio López propiciaron la formación de una planicie aluvial con cursos de agua que fluían en dirección perpendicular hacia el actual canal. Durante el Holoceno temprano (11.500-8.000 años cal AP), la vegetación fue similar a la que se desarrolló en los sectores occidental y central del canal Beagle. Sin embargo, la influencia del ascenso relativo del nivel del mar y la consecuente disponibilidad de humedad ambiental favoreció el desarrollo temprano del bosque de Nothofagus en Punta Moat, y a los 9.500 años cal AP, el paisaje ya presentaba las características de un bosque denso. En el ámbito de la turbera, el aumento en la humedad efectiva propició el desarrollo de turberas mixtas de Sphagnum y plantas en cojín, con elementos florísticos propios de la Tundra Magallánica, por el efecto del spray marino y como consecuencia de la apertura de canales marinos y formación de las islas Picton y Nueva. Hacia los ca. 7.000 años cal AP en Punta Moat se establecen las condiciones paleogeográficas actuales con el desarrollo del Bosque Magallánico Siempreverde y la vegetación de la Tundra Magallánica.
\end{abstract}




\begin{abstract}
Postglacial vegetation reconstruction and its relation with the relative sea-level rise in the $\mathrm{E}$ extreme of Beagle Channel, Tierra del Fuego, Argentina. The coast-line evolution and vegetation from the Last Glacial Maximum (LGM, ca. $25 \mathrm{ka}$ cal AP) until the middle Holocene are reconstructed from a digital terrain model and a postglacial sea level curve and pollen analysis of a Donatia-Astelia cushion bog located in Punta Moat (Moat-2; 545' 50"S, 66 44'10'W; 40 m s.n.m.), east Beagle Channel. The evolution of plant communities at eastern mouth of the Beagle Channel is broadly in line with the regional postglacial vegetation pattern in areas devoid of ice south of Tierra del Fuego. The regional pattern of postglacial vegetation shows steppe followed by forest-steppe and closed Nothofagus forest. Prior 17,700 cal yr BP, the onset of deglaciation in the Beagle Channel in the current study area of Punta Moat and, the removal of alpine paleoglaciers to the headwaters of the valleys and cirques of Sierra Lucio López, led the formation of an alluvial plain with streams flowing in a perpendicular direction to the current channel. During the early Holocene $(11,500-8,000 \mathrm{cal} \mathrm{yr} \mathrm{BP})$, the vegetation was similar to that developed in the western and central sectors of the Beagle Channel. However, the influence of the relative rise of the sea-level and the marine spray-humidity favored the early development of Nothofagus forest in Punta Moat. By 9,500 cal yr BP the landscape showed the physiognomy of a closed forest. Meanwhile on the peat bog, the Sphagnum-cushion plant mixed type prevailed, including floristic elements characteristics of the Magellanic Moorland, due to mineral input from sea spray in coincidence with the opening of channels and the Picton and Nueva islands formation. Towards the $c a$. 7,000 cal yr BP, the Magellanic Moorland and the Subantarctic Evergreen Forest developed when the coast-line configuration was similar than today.
\end{abstract}

Keywords: Palynology, Palaeogeography, Postglacial environments, Beagle Channel, Tierra del Fuego.

\section{Introducción}

La isla Grande de Tierra del Fuego fue englazada en reiteradas oportunidades durante el Pleistoceno. El canal Beagle y los Andes fueguinos fueron ocupados por una red de glaciares de distinta magnitud durante el Último Máximo Glacial (UMG, $c a .25$ ka cal AP, Rabassa, 2008; Kilian and Lamy, 2012). Los glaciares de descarga del manto de hielo de montaña instalado en la cordillera Darwin $(2.000 \mathrm{~m}$ s.n.m., $55^{\circ} \mathrm{S}-69^{\circ} \mathrm{O}$, Fig. 1a) fluyeron siguiendo valles o depresiones preexistentes como el estrecho de Magallanes, la bahía Inútil-San Sebastián, el lago Fagnano y el canal Beagle, alcanzando en algunos casos la plataforma atlántica (Coronato et al., 2004; Rabassa, 2008). Glaciares alpinos originados por el englazamiento de circos y valles de altura en los Andes fueguinos confluían con los glaciares mayores, y generaban una red de englazamiento regional (Coronato, 1995).

El canal Beagle (54.53'S entre los 66 $60^{\circ}$ y los $70^{\circ} \mathrm{W}$, Fig. 1a), ubicado al sur de la isla Grande de Tierra del Fuego, es un valle de origen tectónico (Bujalesky, 2011) que fue completamente ocupado por el hielo durante el UMG (Rabassa et al., 2000).

Durante el UMG, el paleoglaciar Beagle provenía del manto de hielo de montaña de la cordillera Darwin, recibiendo glaciares tributarios desde los circos y valles interiores de los cordones montañosos de ambos márgenes y se extendía hacia el sector este del canal Beagle, hasta alcanzar su posición más externa al este de la localidad de Punta Moat (Rabassa et al., 1990). Las reconstrucciones paleoambientales y paleoecológicas cuaternarias realizadas en el extremo suroriental del archipiélago fueguino son escasas y corresponden a las localidades de Puerto Harberton (Heusser, 1989a, 1990; Pendall et al., 2001; Markgraf and Huber, 2010) y bahía Moat (Heusser, 1995) en Tierra del Fuego, caleta Róbalo (Heusser, 1989b) en isla Navarino (Chile) y bahía Franklin en el sector suroeste de la isla de los Estados (Fig. 1a; Ponce, 2009; Ponce et al., 2011a). Especial interés reviste la localidad de Punta Moat (Fig. 1a y b), porque es allí donde se han observado los arcos morénicos más extensos correspondientes a la máxima extensión del paleoglaciar Beagle, durante el UMG (Rabassa et al., 1990, 2000). De acuerdo con el fechado radiocarbónico de la base de la turbera de Puerto Harberton, distante unos $40 \mathrm{~km}$ al oeste de Punta Moat (Fig. 1a y b), el retroceso del paleoglaciar Beagle desde su posición más externa habría comenzado con anterioridad a los 14.640 años $C^{14}$ AP (17.711 años cal AP, Heusser, 1989b). Sin embargo, el fechado radiocarbónico basal de la turbera bahía Moat (Heusser, 1995) y la del presente trabajo sugieren una edad Holoceno temprano (11.500-8.000 años cal AP) para el inicio de la acumulación de los depósitos de turba en el extremo sudeste del canal Beagle, la cual es posterior al inicio de formación de las turberas situadas al oeste de Punta Moat, ocurrido durante el Tardiglacial (entre el UMG y 11.500 años cal AP) (Heusser, 1989a, 1989b, 1998). 


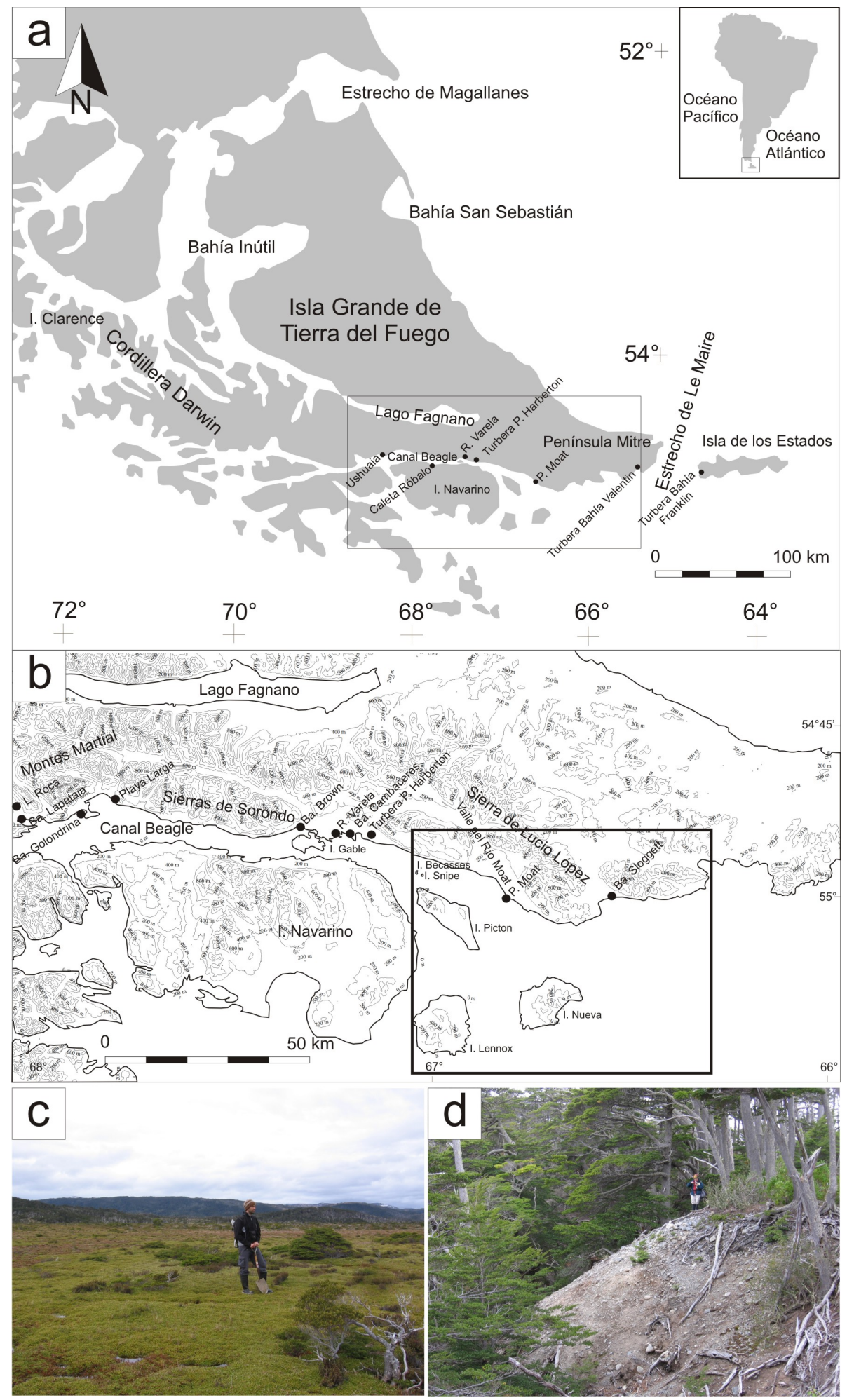

FIG. 1. a. Ubicación; b. mapa topográfico sector sur de Tierra del Fuego y área de estudio delimitada en recuadro; c. turbera Moat 2; d. depósitos glaciofluviales de la planicie sobre la cual se desarrolló la turbera. 
No se conoce aún con certeza cómo ha sido el proceso de deglaciación en el área comprendida entre las localidades de Punta Moat y Puerto Harberton, como tampoco la evolución de las comunidades vegetales que colonizaron dicha área con posterioridad al retiro del hielo. El objetivo del presente trabajo es contribuir al conocimiento de la historia de las comunidades vegetales y su relación con la evolución paleogeográfica del extremo sureste de la isla Grande de Tierra del Fuego con posterioridad al UMG y durante la transición Tardiglacial tardío-Holoceno hasta el Holoceno medio, y al mismo tiempo, establecer la relación entre el ascenso relativo del nivel del mar y el desarrollo de las comunidades del bosque en el área de estudio. Para tal fin se realizó el análisis polínico de la turbera Moat en una nueva perforación, denominada Moat 2, desde la base $(10,0 \mathrm{~m})$ hasta los 4,50 $\mathrm{m}$ de profundidad, y el análisis paleogeográfico del área de Punta Moat (Fig. 1a y b). Este último corresponde a una primera etapa de estudio consistente en el análisis geomorfológico de modelos digitales de terreno. Correlaciones con otros sitios del canal Beagle, isla Navarino e isla de los Estados ayudan a la comprensión de las condiciones paleoclimáticas posglaciales en el extremo sureste del archipiélago fueguino.

\section{Características del área de estudio}

\subsection{Clima y vegetación actuales}

El clima en la porción sureste de la isla Grande de Tierra del Fuego se caracteriza por sus bajas temperaturas, fuertes lluvias y vientos de alta intensidad y frecuencia, provenientes del sur, sudoeste y oeste. Estas condiciones climáticas derivan de su posición latitudinal, de la presencia de un sistema permanente de bajas presiones, de la proximidad a la Corriente Circumpolar del Océano Sur y al continente antártico. De acuerdo con Tuhkanen (1992), se estima para Punta Moat que la temperatura media del mes más frío (julio) varía entre $1^{\circ}$ y $2^{\circ} \mathrm{C}$ y la del mes más cálido (enero), entre $9^{\circ}$ y $10^{\circ} \mathrm{C}$; mientras que las heladas y nevadas pueden ocurrir en cualquier mes del año. $\mathrm{La}$ precipitación media anual excede los $600 \mathrm{~mm}$ (Fritz et al., 2012), observándose un gradiente de precipitaciones oeste-este a lo largo del canal Beagle (Fig. 2), desde la cordillera Darwin $(2.000 \mathrm{~mm})$ hasta Punta Moat $(600 \mathrm{~mm})$ (Tuhkanen, 1992). Entre Lapataia (Fig. 1b) y Punta Moat los valores de precipitación al nivel del mar varían entre $600-500 \mathrm{~mm}$ anuales, pero hacia el este se produce un fuerte incremento del gradiente hasta alcanzar $1.000 \mathrm{~mm}$ anuales en el extremo oriental de la isla Grande de Tierra del Fuego y en la isla de los Estados, como consecuencia del ingreso directo de masas de aire marítimo desde el sur, libre de los obstáculos orográficos que afectan la zona occidental del canal Beagle.

La vegetación del sector sureste del canal Beagle (Fig. 2) está representada por el Bosque Magallánico Siempreverde de Nothofagus betuloides (Mirb.) Oerst ('guindo') o magallánico mixto de $N$. betuloides, $N$. pumilio (Poepp. \& Endl.) Krasser ('lenga') y Drimys winteri J.R. Forst. \& G. Forst ('canelo') (Moore, 1983). Los bosques puros de N.betuloides se pueden observar hacia el interior de Tierra del Fuego, mientras que Drimys winteri comienza a ser codominante en las áreas costeras. El estrato arbustivo y herbáceo se presenta pobre y mal desarrollado. Entre los arbustos asociados se incluyen Empetrum rubrum Vahl ex Willd., y Philesia magellanica Gmel., localmente subdominantes en las áreas abiertas del bosque. Entre los asociados en el estrato arbustivo figuran Berberis ilicifolia L.f., Blechnum magellanicum (Desv.) Mett. y en el estrato herbáceo aparecen Blechnum pennamarina (Poiret) Kuhn, Gunnera magellanica Lam., Acaena ovalifolia Ruiz \& Pavón, Hymenophyllum spp., Luzuriaga marginata (Gaertn.) Benth \& Hooker y Senecio acanthifolius Hombron \& Jacquinot. Sobre las márgenes del Bosque Magallánico Siempreverde, y hacia el interior de Tierra del Fuego, se encuentran una serie de comunidades de pantanos turbosos que colectivamente han sido denominadas como la Tundra Magallánica (Pisano, 1977) o 'Magellanic Moorland' (Moore, 1983). Constituye un paisaje sin árboles, con gran desarrollo de pantanos turbosos con matorrales dominados por la asociación de Donatia fascicularis Forst., que forman pequeños cojines y Astelia pumila (Forster f.) Gaudich constituyendo densos céspedes. Entre sus asociados aparecen Caltha dioneifolia Hooker, Drapetes muscosus Banks ex Lam., Tetroncium magellanicum Willd., Schoenus antarcticus (Hooker f.) Dusén, Gaimardia australis Gaudich., Uncinia kingii Boott, Acaena pumila Vahl, Drosera uniflora Willd., Gaultheria antarctica Hooker, Gunnera lobata Hooker, Marsippospermum grandiflorum (L.f.) Hooker f., Myrteola nummularia (Poiret) Berg, Nanodea muscosa Banks ex C.F. Gaertner, Perezia magellanica (L.f.) Lag., Pernettya pumila (L.F.) Hooker, Tapeinia pumila (Forster f.) Baillon 


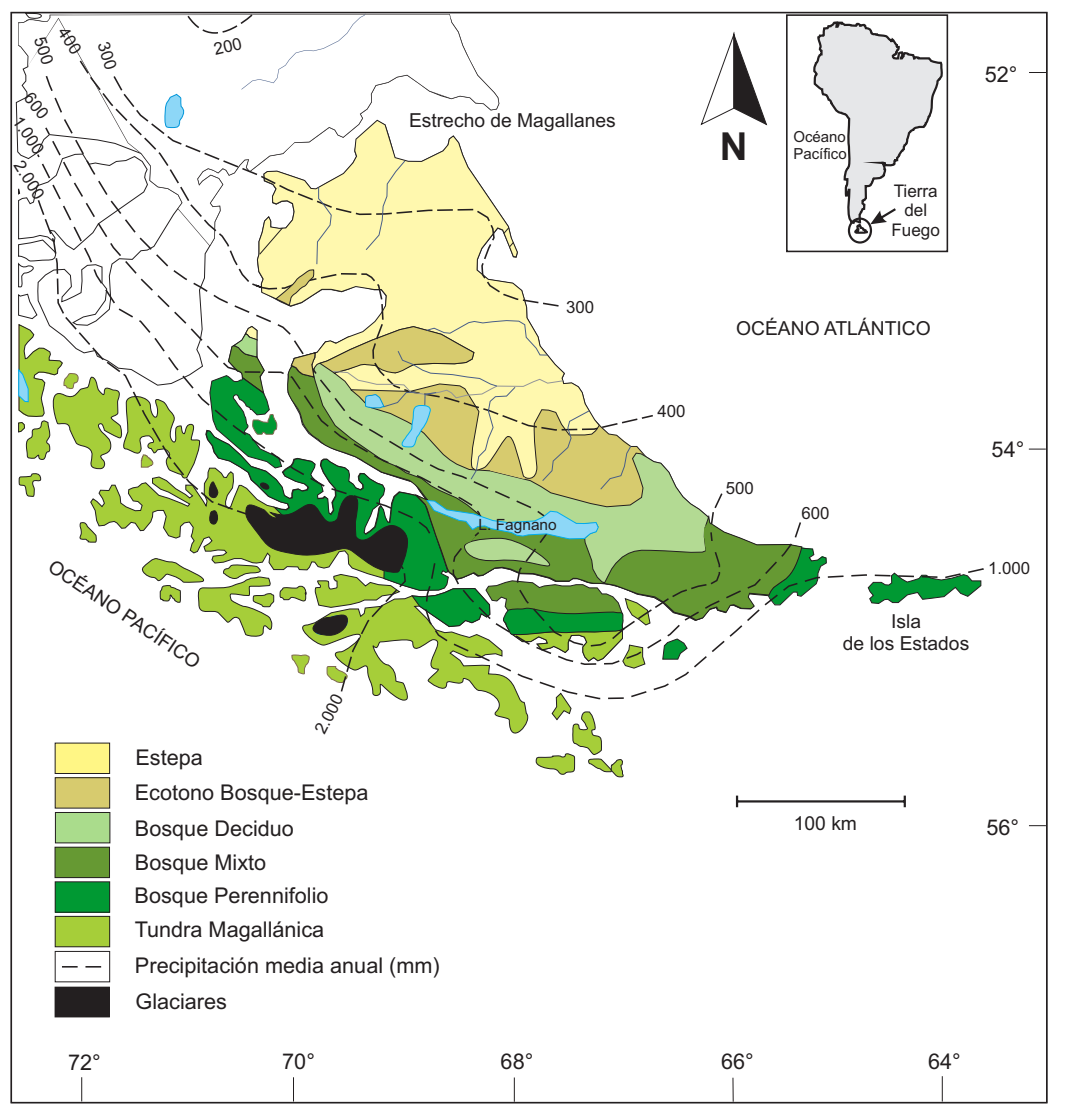

FIG. 2. Mapa de unidades fitogeográficas (modificado de Tuhkanen, 1989,1990) y curvas de precipitación anual (mm).

y Tribelis australis Phil. Entre los arbustos se encuentran Berberis ilicifolia, Chiliotrichium diffusum (Forster f.) O. Kuntze, Embotrhium coccineum Forster \& Forster, Empetrum rubrum, Escallonia serrata Sm. y Philesia magellanica Gmel. que a menudo desarrollan comunidades transicionales entre el bosque y el pantano (Moore, 1983).

\subsection{Localidad Punta Moat}

La turbera estudiada, Moat $2\left(54^{\circ} 58^{\prime} 50^{\prime}\right.$ 'S, $66^{\circ} 44^{\prime}$ $10^{\prime} \mathrm{W}, 40 \mathrm{~m}$ s.n.m.), se encuentra ubicada al este del río Moat, a menos de $1 \mathrm{~km}$ del canal Moat y distante unos $100 \mathrm{~km}$ al este de la ciudad de Ushuaia (Fig. 1a). Cubre una extensión de $1,7 \mathrm{~km}^{2}$ y se encuentra rodeada por el Bosque Magallánico Siempreverde de Nothofagus betuloides y Drimys winteri (Fig.1c). La superficie de la turbera se caracteriza por un tapiz herbáceo bajo, denso y compacto, formado en su mayor parte por cojines de Donatia fascicularis y céspedes de Astelia pumila que le imprimen un aspecto irregular de microrrelieves interrumpidos por numerosas charcas de agua dulce (Pisano, 1977). Estos ambientes fueron caracterizados como turberas en carpeta (Tundra Magallánica), típicos de la región oriental y más lluviosa de Tierra del Fuego (Roig y Collado, 2004). En la isla Grande de Tierra del Fuego, este tipo de turberas se desarrollan en cercanías a las costas, mientras que tierra adentro son gradualmente reemplazadas por turberas elevadas de Sphagnum magellanicum, las cuales existen bajo condiciones de mayor continentalidad (Kleinebecker et al., 2007).

\section{Materiales y métodos}

La sección fósil estudiada, denominada Moat 2, se extrajo con una sonda rusa, con sacatestigo de $50 \mathrm{~cm}$ de longitud. Previo al muestreo, se realizaron perforaciones en toda la turbera con una equidistancia de 30 a $40 \mathrm{~m}$ para determinar el sector de máxima 
profundidad donde obtener el testigo. La sección estudiada presenta $10 \mathrm{~m}$ de longitud y corresponde a la máxima profundidad alcanzada. Se inicia con un nivel de $5 \mathrm{~cm}$ de arcillas orgánicas grises oscuras, seguidas de turba homogénea, entre los 2,60-1,40 m $\mathrm{y}$ durante los últimos $0,50 \mathrm{~m}$ de profundidad se observan intercalaciones de turba fibrosa.

Para el análisis polínico se estudiaron un total de 109 muestras desde la base hasta la profundidad de $4,50 \mathrm{~m}$, a intervalos de $3 \mathrm{~cm}$. Cada muestra palinológica comprende $1 \mathrm{~cm}$ de espesor. Las muestras fueron procesadas de acuerdo con las técnicas de Faegri e Iversen (1989). A cada muestra se le agregaron tabletas de esporas de Lycopodium clavatum para el cálculo de la concentración polínica por gramo de sedimento seco y para el cálculo de la tasa de acumulación de polen (influjo polínico) por gramo de sedimento seco por año (Stockmarr, 1971). Las frecuencias relativas (\%) de árboles, arbustos y hierbas de origen terrestre fueron calculadas a partir de sumas totales superiores a 300 granos de polen. Las frecuencias relativas de polen de plantas acuáticas y criptógamas se calcularon sobre la suma total de polen y esporas contados. La zonación palinológica del perfil fósil fue realizada mediante 'Cluster Analysis' (CA) Constrained single-link (Birks y Gordon, 1985), utilizando como coeficiente de disimilitud la distancia de Edwards y Cavalli-Sforza (Overperck et al., 1985; Prentice, 1986). Los diagramas de las frecuencias relativas y de la tasa de acumulación de polen y el análisis de agrupamiento se realizaron mediante el programa Tilia y Tiliagraph (TGview 2.0.2, Grimm, 2004). Las especies arbóreas de Nothofagus betuloides, $N$. pumilio y $N$. antarctica fueron incluidas dentro del grupo taxonómico Nothofagus tipo dombeyi dado que los granos de polen poseen una morfología similar que dificulta su diferenciación. En 'Otros' se incluyen aquellos taxa con baja representación $(<1 \%)$ como Caryophyllaceae, Rubiaceae, Escallonia, Berberis, Pratia, Brassicaceae, Asteraceae subf. Cichorioideae, Chenopodiaceae, Ribes y Plantago. Las asociaciones polínicas fósiles se compararon por analogía con las actuales registradas en muestras superficiales de Tierra del Fuego (Heusser, 1989b; Trivi de Mandri et al., 2006; Musotto et al., 2012) y de la isla de los Estados (Ponce et al., 2011a). Las comunidades vegetales fósiles se compararon con unidades de vegetación actual (Pisano, 1977; Moore, 1983).
Para la elaboración del modelo paleogeográfico usamos el programa Global Mapper $10^{\circledR}$ para la generación de un modelo digital de ascenso del nivel del mar, teniendo en cuenta la curva de ascenso del nivel del mar propuesta por Fleming et al. (1998) desde la Última Glaciación hasta la actualidad. Con este programa fueron analizados los modelos digitales de elevación del terreno de la Shuttle Radar Topography Mission (SRTM), W100S10.Bathymetry.SRTM y W060S10.Bathymetry.SRTM., con un pixel de 1x1 km de resolución espacial (http:// topex.ucsd.edu/WWW_html/srtm30_plus.html). Con estas herramientas fueron elaborados sucesivos mapas paleogeográficos, que muestran la posición del nivel del mar, con un intervalo de variación de $10 \mathrm{~m}$, comenzando a los 12.000 años cal A.P. y finalizando a los 9.000 años cal A.P. El tiempo aproximado para cada una de las diferentes posiciones del nivel del mar fue obtenido de la curva de Fleming et al. (1998). Las edades calibradas indicadas se lograron de los datos presentados en Fleming et al. (1998). No se ha tenido en cuenta para la elaboración del modelo la acción erosiva de las corrientes marinas sobre los sedimentos depositados en el fondo marino ni la acción tectónica.

Para el análisis geomorfológico se utilizó el programa Global Mapper 10 con los modelos digitales de elevación del terreno del sensor Shuttle Radar Topography Mission (SRTM), S56W067 y S55W067 con un pixel de 90x90 m de resolución (https://lta. cr.usgs.gov/SRTM2). Con este programa se realizaron mediciones altimétricas y planimétricas de las geoformas mapeadas y se elaboró una base topográfica.

Para el mapeo geomorfológico también se realizó fotointerpretación de pares estereoscópicos del área de estudio a escala 1:40.000 tomados por el Servicio de Hidrografía Naval en el año 1970. El trabajo de gabinete fue complementado con tareas de campo que consistieron en la identificación, descripción y mapeo de depósitos y geoformas glacigénicas, siguiendo las pautas metodológicas habituales en los estudios de Geología del Cuaternario y Geomorfología.

Las dataciones radiocarbónicas sobre turba (Tabla 1) fueron realizadas en el Institute of Accelerator Analysis Ltd. (IAA), Japan. La calibración de las edades radiocarbónicas se realizó con el programa Calib Rev 5.1.0 (Stuiver et al., 2005), curva de calibración del Hemisferio Sur (SHcal04.14c; McCormac et al., 2004). Para el modelo de edad-profundidad (Fig. 3) se utilizó la curva polinomial de segundo grado. 
TABLA 1. DATACIONES RADIOCARBÓNICAS Y EDADES CALIBRADAS, SITIO MOAT 2.

\begin{tabular}{|c|c|c|c|c|c|c|c|}
\hline $\begin{array}{l}\text { Profundidad } \\
\text { (cm) }\end{array}$ & Edad ${ }^{14} \mathrm{C}$ AP & $\begin{array}{c}\text { Edad calendario } \\
\text { AP }\end{array}$ & Máximo $2 \sigma \mathrm{BP}$ & Mínimo $2 \sigma \mathrm{BP}$ & $\delta^{13} \mathrm{C} \%$ & Material & $\begin{array}{c}\text { Número } \\
\text { laboratorio }\end{array}$ \\
\hline 452 & $4.533 \pm 39$ & 5.146 & 5.301 & 4.969 & $-24,9$ & turba & IAAA-75401 \\
\hline 700 & $5.220 \pm 40$ & 5.923 & 6.001 & 5.854 & $-24,5$ & turba & IAAA-52277 \\
\hline 752 & $6.040 \pm 40$ & 6.823 & 6.944 & 6.717 & $-28,27$ & turba & IAAA-71698 \\
\hline 950 & $8.710 \pm 40$ & 9.608 & 9.704 & 9.533 & $-29,7$ & turba & IAAA-52278 \\
\hline 996 & $9.750 \pm 40$ & 11.195 & 11.241 & 11.122 & $-25,9$ & turba & IAAA-52279 \\
\hline
\end{tabular}

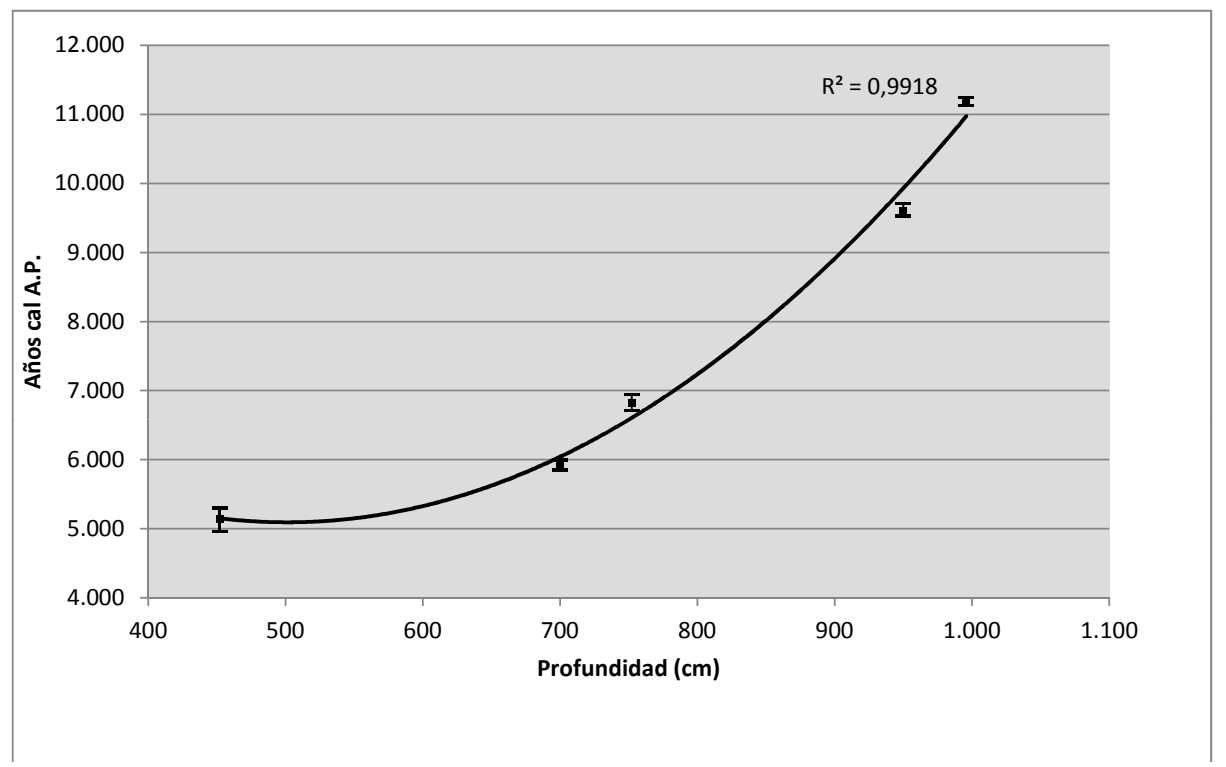

FIG. 3. Curva edad versus profundidad, sitio Moat 2.

\section{Resultados}

\subsection{Palinología}

En la secuencia fósil, el análisis de agrupamiento $\mathrm{y}$ análisis visual permitieron reconocer tres zonas polínicas (Fig. 4). Los valores de porcentaje para cada taxón se expresaron como valores mínimo, promedio, indicado entre paréntesis, y máximo.

Zona M-1 (1.000-950 $\mathrm{cm}$ de profundidad, 11.200-9.500 años cal AP): se caracteriza por Nothofagus tipo dombeyi $(8,3-(25,5)-62,2 \%)$, Gunnera (2,1-(21,6)-49,3\%), Asteraceae subf. Asteroideae (0,3-(15,6)-35,6\%), Poaceae (7,4-(15,5)-30,1\%) y Empetrum rubrum $(0,3-(11,7)-25,9 \%)$. El registro de Cyperaceae varía entre 0,3-(3,7)-13,0\%. En la parte inferior de la zona, entre los 11.200 y
10.500 años cal AP, Gunnera (3,8-(26,2)-49,3\%), Asteraceae subf. Asteroideae (12,2-(25,1)-35,6\%), Poaceae (11,3-(19,4)-30,1\%) y Empetrum rubrum (2,0-(13,6)-25,9\%) muestran valores altos, mientras Nothofagus tipo dombeyi $(8,3-(10,5)-15,7 \%)$ registra su valor más bajo de toda la secuencia. Después de los 10.500 años cal AP, se incrementa el polen de Nothofagus $(22,4-(38,7)-62,2 \%)$. Esta zona se caracteriza por valores de influjo polínico bajos en comparación con los registrados en el resto de la secuencia (Fig. 5). El influjo promedia unos 29.200 granos/gramo/año (8.600-44.000 granos/gramo/ año) con un máximo de 152.700 granos/gramo/año a los $956 \mathrm{~cm}$ de profundidad. Los arbustos y hierbas registran valores de influjo polínico más altos que los árboles y plantas acuáticas. El tiempo de depositación es alto (33,2 años/gramo de sedimento). 


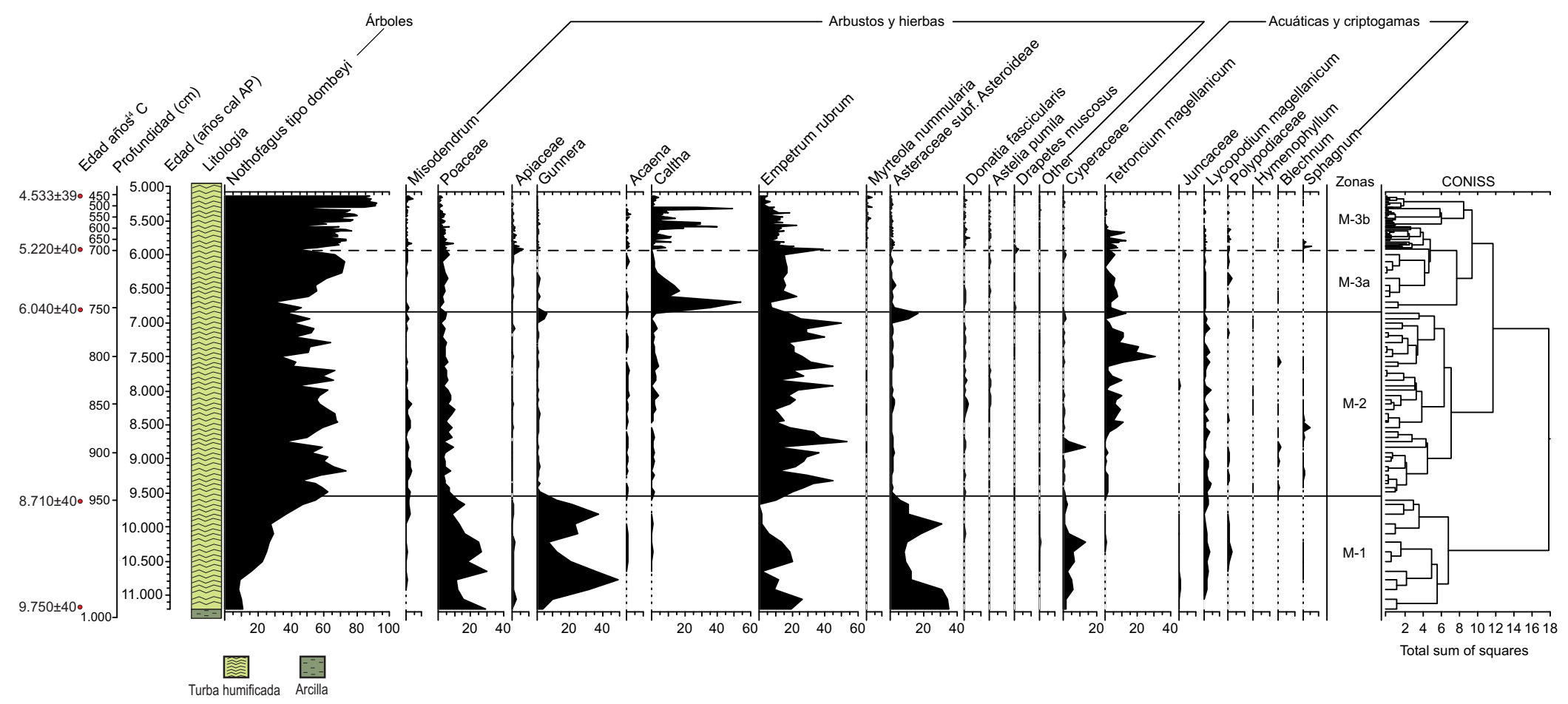

FIG. 4. Diagrama polínico de frecuencias relativas, sitio Moat 2. 


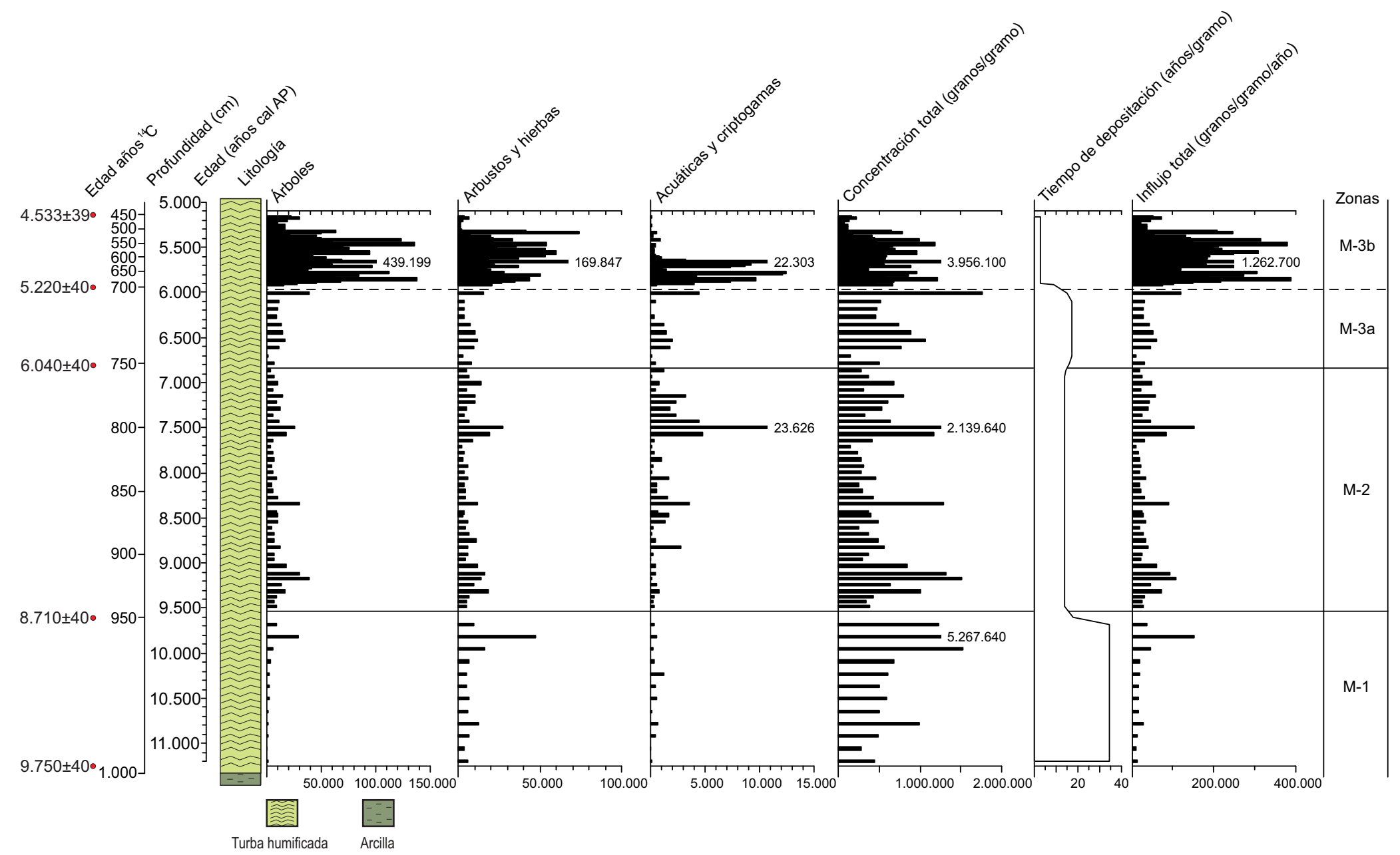

FIG. 5. Diagrama de influjo polínico, sitio Moat 2. 
Zona M-2 (950-750 $\mathrm{cm}$ de profundidad, 9.5006.800 años cal AP): está caracterizada por Nothofagus tipo dombeyi (33,1-(54,8)-73,4\%), Empetrum rubrum $(9,0-(26,3)-53,2 \%)$ y Poaceae $(2,1-(5,6)-10,8 \%)$. Misodendrum registra 0,2-(1,3)-3,4\%. Gunnera, Asteraceae subf. Asteroideae y Cyperaceae están presentes con valores bajos $(<1,7 \%)$. Tetroncium magellanicum alcanza 0,2-(6,1)-30,9\%. El influjo polínico total muestra un incremento que promedia 40.560 granos/gramo/año, con un máximo de 152.100 granos/gramo/año a los $800 \mathrm{~cm}$ de profundidad. El tiempo de depositación disminuye (14,1 años/gramo de sedimento).

Zona M-3 (750-450 $\mathrm{cm}$ de profundidad, 6.8005.000 años cal AP) se dividió en dos subzonas:

- Subzona M-3a (750-700 cm de profundidad, 6.800-6.000 años cal AP): se caracteriza por un paulatino aumento de Nothofagus tipo dombeyi $(29,2-(57,9)-72,6 \%)$ hacia el final de la subzona. Caltha alcanza valores altos de porcentaje (hasta $54,2 \%$ ) al inicio de la subzona y luego disminuye $(1 \%)$ hacia el final de la subzona. Empetrum rubrum (7,2-(14,9)-22,9\%), Poaceae (1,3-(4,1)$7,2 \%)$ y Tetroncium magellanicum $(2,6-(4,4)-$ $8,0 \%$ ) muestran una disminución en sus valores con respecto a la zona anterior (M-2). El influjo polínico total promedia 44.000 granos/gramo/año y el tiempo de depositación aumenta a 17,3 años/ gramo de sedimento.

- Subzona M-3b (700-450 cm de profundidad, 6.0005.000 años cal AP): caracterizada por el dominio de Nothofagus tipo dombeyi $(40,1-(69,8)-91,8 \%)$. Caltha muestra valores de porcentaje variables con dos máximos (39,6 y 49,2\%). Poaceae (0,3-(3,2)9,2\%) y Empetrum rubrum (2,6-(11,0)-39,0\%) disminuyen con respecto a la subzona anterior, mientras aumenta el polen de Misodendrum (0,3$(0,9)-4,2 \%)$. El influjo polínico total se incrementa abruptamente, con un promedio de 175.600 granos/ gramo/año y luego disminuye hacia el final de la subzona con un promedio de 35.800 granos/gramo/ año. El tiempo de depositación también disminuye a 3,1 años/gramo de sedimento.

\subsection{Análisis geomorfológico}

El área de estudio se encuentra localizada en la confluencia de dos antiguos valles glaciarios: aquel del glaciar Beagle, el cual fluía en dirección oesteeste por la artesa principal, y el del glaciar Moat, que fluía con una dirección general oeste-este y noroestesudeste en su tramo inferior, por el valle homónimo formando una artesa independiente (Fig. 1b). En la zona de confluencia de ambos paleoglaciares se desarrolló un campo de drumlins (Fig. 6). Este campo limita al norte y noreste con las paredes rocosas del tramo inferior del valle del río Moat, cuya pendiente es de $15^{\circ}$, y hacia el sur y suroeste con el mar. Está compuesto por 95 drumlins distribuidos en un área de aproximadamente $80 \mathrm{~km}^{2}$. Dichas geoformas desaparecen abruptamente hacia el sur, en donde son interceptadas por la actual línea de costa formando en algunos casos acantilados de hasta $50 \mathrm{~m}$ de altura; probablemente, parte del campo de drumlins se encuentre sumergido o haya sido erosionado por acción marina. Los drumlins son elongados en dirección $\operatorname{SSE}\left(\mathrm{N} 150^{\circ}\right)$. La extensión longitudinal de estas geoformas varía entre 290 y $620 \mathrm{~m}$, la altura promedio es de 20-25 $\mathrm{m}$ y el ancho promedio de la base es cercano a los $210 \mathrm{~m}$. La mayoría de los drumlins se encuentran cubiertos por un bosque denso de Nothofagus betuloides y las áreas interdrumlins por extensas turberas con vegetación en cojín dominadas por Donatia fascicularis y Astelia pumila (Fig. 1c). Paralelas a la costa actual se extienden las morrenas fronto-laterales. Estas geoformas se desarrollan entre los 90 y 10 m s.n.m. El campo de drumlins y las morrenas son atravesados por el río Moat, el cual presenta una dirección general NNE-SSO (Fig. 6); en algunos sectores de su tramo inferior tiene un encajonamiento sobre el lecho rocoso del orden de los $25 \mathrm{~m}$. No se han observado en el área de estudio terrazas marinas que sugieran un levantamiento tectónico y/o glaciotectónico.

El testigo analizado (Moat 2) fue obtenido de una turbera en carpeta dominada por Donatia fascicularis y Astelia pumila, ubicada en el interior del campo de drumlins. La turbera se desarrolla sobre una antigua planicie glacifluvial que limita hacia el este con el río Moat y hacia el sur con una morrena fronto-lateral del paleoglaciar Beagle (Fig. 6). La pendiente general de esta planicie es del orden de los $0,6^{\circ}$, hacia el SE. En algunos sectores de las barrancas del río Moat, se exponen depósitos glacifluviales de 1,5 $\mathrm{m}$ de espesor, compuestos por gravas y arenas con estructura cruzada (Fig. 1d). Esta planicie glacifluvial presenta una serie de terrazas con rumbo norte-sur, escalonadas hacia el oeste a diferentes niveles: 33,1 m s.n.m, 35,5 m s.n.m. y 


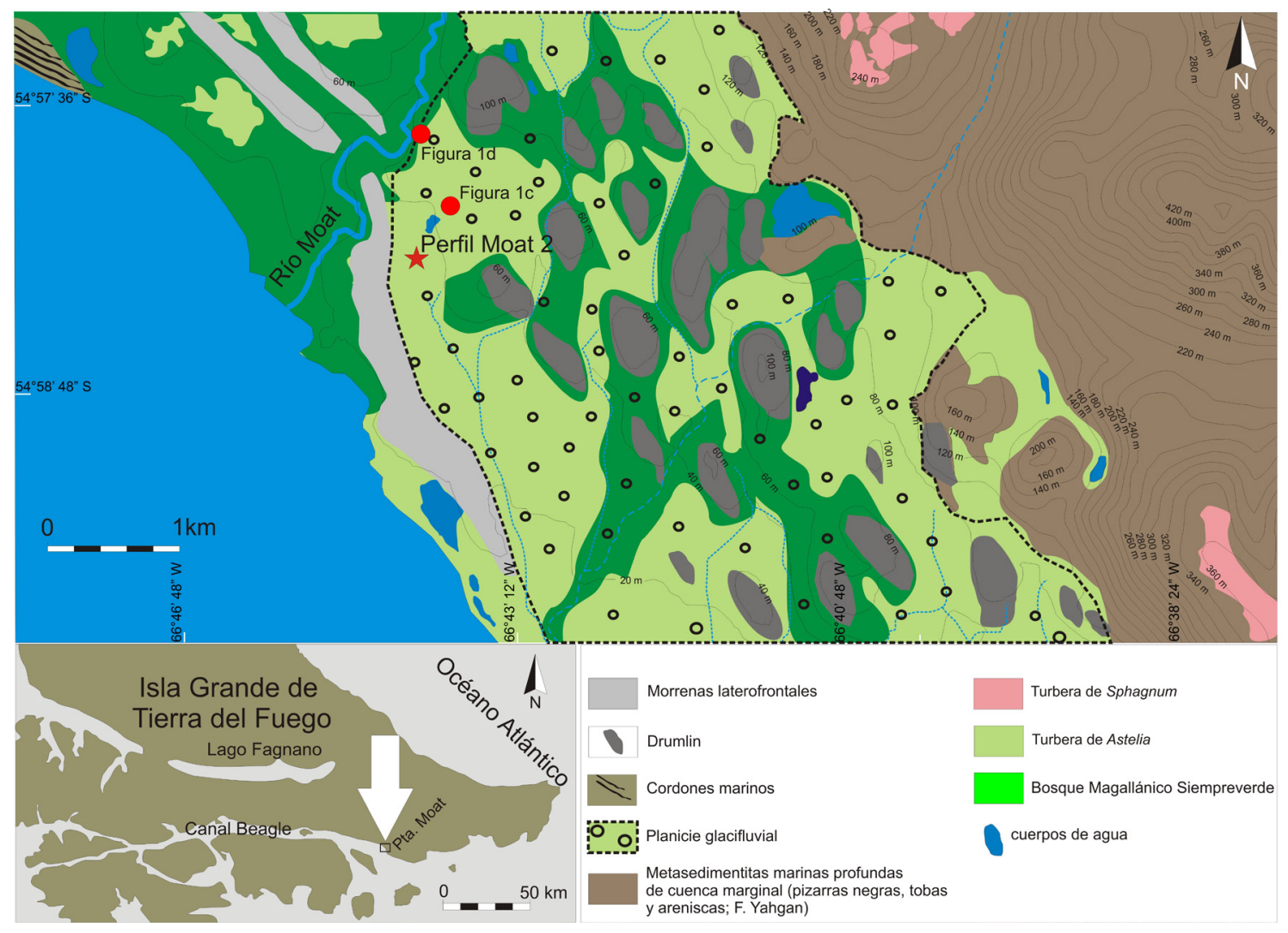

FIG. 6. Mapa geomorfológico del área de Punta Moat.

40,3 m s.n.m. y sobre cada una de ellas se desarrollan las comunidades vegetales que forman la turbera.

\section{Discusión}

\subsection{Modelo paleogeográfico}

Playas elevadas de edad Holocena han sido reconocidas sobre la costa norte del canal Beagle, únicamente en el centro y oeste entre las localidades de bahía Lapataia-lago Roca y bahía Cambaceres, al oeste del área de estudio (Fig. 1b; Rabassa et al., 1986; Gordillo et al., 1992; Isla y Bujalesky, 2008). Las playas elevadas más antiguas presentan una edad cercana a los 6.000 años AP, y las alturas de estas varían desde un máximo de 8-10 m en el oeste: lago Acigami -Roca-, bahía Golondrina, playa Larga (Rabassa et al., 1986; Rabassa, 1987; Gordillo et al., 1992, 1993; Bujalesky, 1998; Bujalesky, 2007) disminuyendo hacia el este a alturas cercanas a los
$6 \mathrm{~m}$ en bahía Cambaceres (Zangrando et al., 2009) (Fig. 1b). Hacia el este de esta última localidad, no han sido descritas hasta el momento playas elevadas holocenas. Mörner $(1987,1991)$ señaló que el estrecho de Magallanes y canal Beagle han sido sometidos a diferentes comportamientos de levantamiento y que en términos generales la zona no ha sido sometida a una significativa acción glacioisostática durante el Holoceno. Rabassa et al. (1986) propusieron un levantamiento promedio de 1,5 a 2,0 mm/año para el área de canal Beagle, como resultado de la combinación de levantamiento tectónico y glacioisostacia. Isla y Bujalesky (2008) indican que el levantamiento tectónico durante los últimos 8.000 años fue mayor hacia el oeste del canal (aproximadamente 1,2 $\pm 0,2 \mathrm{~mm} / \mathrm{año}$ ), disminuyendo hacia el este, siendo aparentemente despreciable en la costa norte de Tierra del Fuego e indican que el rebote glacioisostático parece haber operado durante la deglaciación o durante los mil o 2 mil años que 

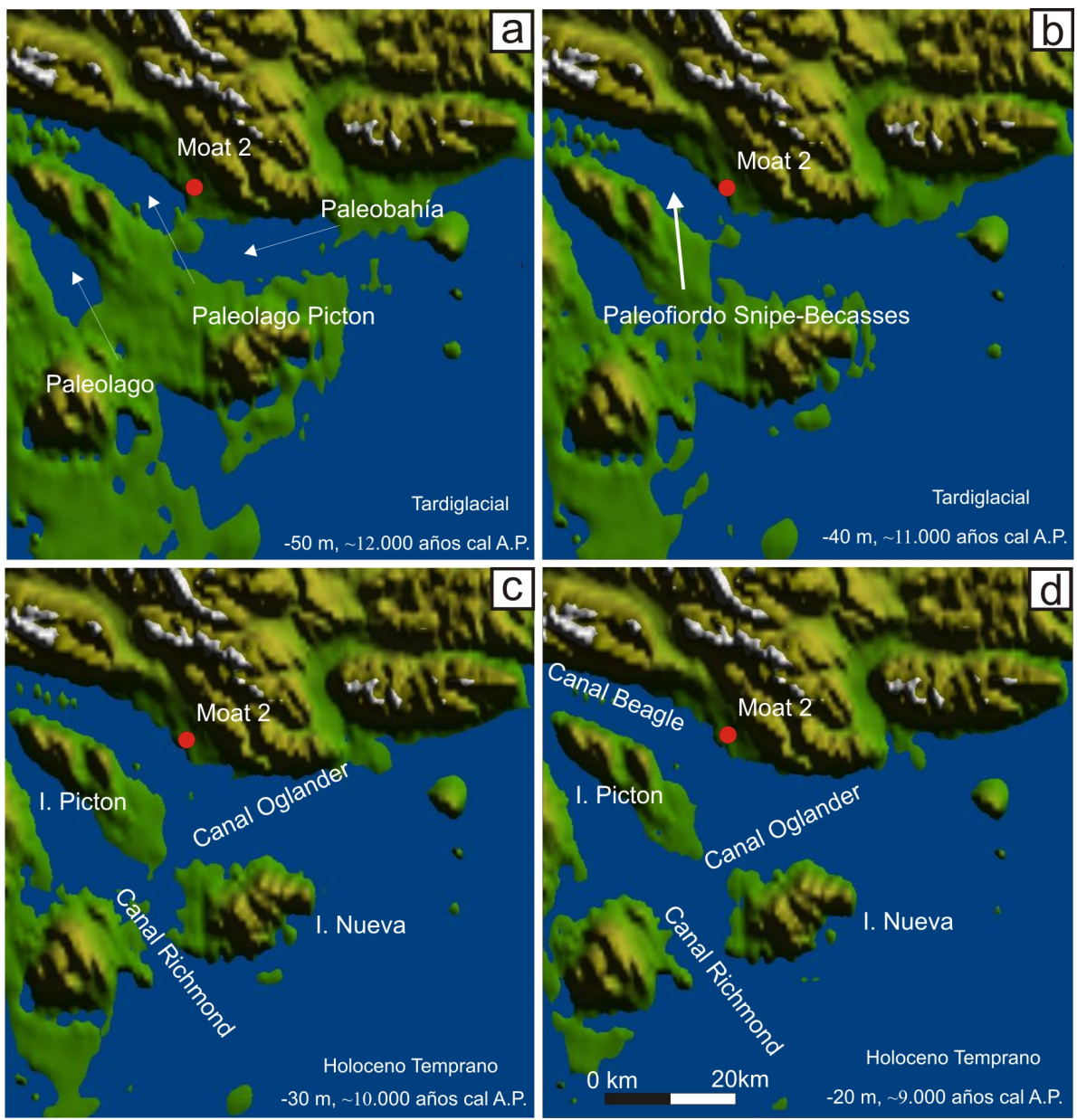

FIG. 7. Evolución paleogeográfica del área de estudio. a. 12.000 años cal AP; b. 11.000 años cal AP; c. 10.000 años cal AP; d. 9.000 años cal AP.

la precedieron. Sobre la base de las cotas de playas elevadas a lo largo del canal Beagle, Bujalesky (2011) propone la existencia de un levantamiento promedio para el canal del orden de $1,3 \mathrm{~mm} /$ año durante los últimos 6.000 años. Es evidente que estos valores de levantamiento no son representativos para todo el canal Beagle, ya que, como se indicó anteriormente, las mayores diferencias de cotas se observan en el sector oeste del canal y disminuyen notoriamente hacia el este, hasta desaparecer en el área de estudio. Por lo anteriormente expuesto, y dado que hasta el momento no se han encontrado evidencias de campo que indiquen movimientos tectónicos y/o glacioisostáticos en el área de estudio, el modelo paleogeográfico propuesto se basa en batimetría ajustada a la curva de ascenso global del nivel del mar propuesto por Fleming et al. (1998). De esta manera, hacia los 12.000 años cal AP, momento cercano al inicio de formación de la turbera aquí estudiada, la superficie de tierra emergida en la boca oriental del canal Beagle se habría reducido a casi un tercio $\left(11.500 \mathrm{~km}^{2}\right)$ de su tamaño durante el UMG. Hacia el sur de Punta Moat, aún se mantenían unidas entre sí las actuales islas Navarino, Picton, Lennox y Nueva y a la isla Grande de Tierra del Fuego. Asimismo, una extensa bahía se habría desarrollado al este de Punta Moat, adyacente a la actual isla Nueva, mientras que hacia el oeste se habría formado un lago de $120 \mathrm{~km}^{2}$ de superficie (Fig. 7a), a juzgar por la existencia de una depresión topográfica o paleolago que denominamos paleolago Picton. 
Hacia los 11.000 años cal AP, con el ascenso relativo del nivel de mar hasta los $-40 \mathrm{~m}$, se habría producido el ingreso de agua marina en el paleolago Picton y la inundación de la porción de tierra que lo separaba de la bahía ubicada al este de Punta Moat (Fig. 7b).

La separación de las islas Nueva y Picton entre sí y del resto de la isla Grande de Tierra del Fuego se habría producido hacia los 10.000 años cal AP aproximadamente, cuando el nivel del mar alcanzó los $-30 \mathrm{~m}$, profundidad máxima de los tres canales que hoy separan dichas islas (Fig. 7c). Para este momento, nuestro modelo paleogeográfico muestra que ambas islas tenían una superficie que duplicaba su superficie actual y que la isla Lennox ya se encontraba separada de ellas, pero aún unida a la actual isla Navarino.

Hacia los 9.000 años cal AP, cuando el nivel del mar global alcanzó los -20 m, las costas en el área de estudio adquieren una configuración geográfica similar a la actual (Fig. 7d). Esta se habría alcanzado hacia los 7.000 años cal AP.

\subsection{Reconstrucción de las comunidades vegetales en Punta Moat}

La reconstrucción de la historia de la vegetación permite observar entre los 11.200 y 10.500 años cal AP, el predominio de gramíneas (Poaceae), hierbas (Gunnera), arbustos (Asteraceae subf. Asteroideae) y murtillares (Empetrum rubrum) que sugieren el desarrollo de praderas graminosas junto con matorrales y arbustos. Dichas comunidades vegetales serían análogas a las que se desarrollan actualmente en la estepa del norte-centro de Tierra del Fuego (Fig. 2), donde las precipitaciones son inferiores a los $400 \mathrm{~mm}$ anuales y las temperaturas medias estivales de $10^{\circ}$ $11^{\circ} \mathrm{C}$ (Pisano, 1977; Heusser, 1989b). La abundancia de ciperáceas en el depósito sugiere la existencia de condiciones minerotróficas durante el desarrollo de la turbera. El registro de Lycopodium magellanicum se asocia con las partes más secas de la turbera como también con las comunidades abiertas de pastizales y arbustos (Moore, 1983), estando este mejor representado durante las zonas polínicas M-1 y M-2. En el inicio de la secuencia, el polen de Nothofagus registra valores inferiores al $15 \%$, valor semejante al de los espectros polínicos de muestras superficiales de la estepa fueguina (Heusser, 1989b; Musotto et al., 2012), que sugieren un transporte aéreo extrarregional
( $>200 \mathrm{~km}$ de distancia desde la fuente emisora sensu Prentice, 1985). A partir de los 10.800 años cal AP el incremento de Nothofagus es acompañado por el registro de Misodendrum, género hemiparásito de Nothofagus. En muestras de superficie ubicadas en la estepa y ecotono bosque-estepa del norte-centro y centro de Tierra del Fuego, respectivamente, las frecuencias bajas de Nothofagus y de Misodendrum mostrarían el establecimiento regional (entre 2 y $200 \mathrm{~km}$ de distancia desde la fuente emisora, sensu Prentice, 1985) del bosque de Nothofagus (Musotto et al., 2012). Entre los 10.500 y 9.500 años cal AP, el aumento gradual en los valores de porcentaje de Nothofagus acompañado por proporciones aún elevadas de Poaceae, Gunnera y Asteraceae subf. Asteroideae sugieren la presencia de comunidades vegetales del ecotono bosque-estepa similares a las que se desarrollan actualmente en el centro de Tierra del Fuego (Fig. 2; Musotto et al., 2012) donde las precipitaciones alcanzan valores superiores a los $400 \mathrm{~mm}$ anuales.

A partir de los 9.500 años cal AP, el espectro polínico refleja un cambio sustancial en la vegetación del área y de la turbera. Las mayores proporciones relativas de Nothofagus y la disminución de Poaceae, Gunnera y Asteraceae subf. Asteroideae sugieren el predominio de las comunidades del bosque sobre las del ecotono bosque-estepa. Las condiciones paleoclimáticas habrían sido análogas a las del Bosque Magallánico Deciduo del centro-sur de Tierra del Fuego (Fig. 2), donde las precipitaciones oscilan entre los 450 y $600 \mathrm{~mm}$ anuales y la temperatura del mes más cálido es de $9{ }^{\circ} \mathrm{C}$ (Pisano, 1977). El aumento en las frecuencias relativas de Nothofagus junto con el registro de Misodendrum indican la presencia del bosque en el área. En muestras de superficie ubicadas en el bosque abierto, se registraron valores porcentuales altos de Nothofagus $(>50 \%)$ y bajos de Misodendrum ( $<5 \%$ ) coincidentes con una ubicación extralocal del bosque $(<2 \mathrm{~km}$ de distancia desde la fuente emisora, sensu Prentice, 1985; en Musotto et al., 2012). La disminución de Cyperaceae y el incremento de Empetrum rubrum, especie común en las turberas de Sphagnum que coloniza las partes elevadas ('hummocks') y más secas sugieren un cambio en las condiciones tróficas de la turbera hacia la ombrotrofía (Heusser, 1998). Por otro lado, la presencia de Astelia pumila, Donatia fascicularis y Drapetes muscosus, elementos característicos de la tundra hiperhúmeda, con valores 
bajos de frecuencias, sugieren posiblemente un estadío transicional entre la turbera elevada de Sphagnum y la típica turbera en carpeta (Tundra Magallánica). Actualmente, en el sur de Chile $\left(52^{\circ} \mathrm{S}\right)$ se observa en las áreas costeras, el desarrollo de turberas mixtas transicionales de Sphagnum y plantas en cojín (Kleinebecker et al., 2007). Asimismo, el registro de Tetroncium magellanicum, típico elemento de la Tundra Magallánica, indica la presencia de charcas de agua en la turbera, dado que esta especie es capaz de soportar largos períodos de inmersión en el agua. Durante este intervalo, el incremento en los valores de influjo polínico y la disminución en el tiempo de depositación (Fig. 5) señalan una tasa de crecimiento del depósito positiva probablemente relacionada con condiciones de mayor humedad y menor temperatura.

Con posterioridad a los 6.800 años cal AP, las comunidades del bosque de Nothofagus continúan dominando el paisaje. La presencia de Poaceae se relaciona con el desarrollo de praderas graminosas en el entorno del depósito. Estas comunidades graminoideas actualmente crecen donde hay escurrimiento superficial de agua (Moore, 1983). Asimismo, el notable incremento de Caltha y la disminución de Empetrum rubrum indicarían niveles más altos de humedad en la turbera. Caltha, al igual que Tetroncium magellanicum, son especies higrófilas y junto con la vegetación de Astelia pumila, Donatia fascicularis y Drapetes muscosus, se desarrollan en aquellos ambientes en contacto con el Bosque Magallánico Siempreverde en el sudeste de la isla (Fig. 2; Moore, 1983). Las condiciones paleoambientales serían análogas a estas comunidades vegetales y sugieren valores de precipitación del orden de los $800 \mathrm{~mm}$ anuales (Heusser, 1998).

Entre los 6.000 y ca. 5.000 años cal AP, si bien el registro polínico refleja la persistencia del bosque de Nothofagus, el mismo habría sido mucho más denso que anteriormente, como lo indican el incremento en los valores de concentración e influjo polínico (Fig. 5). Actualmente se han registrado valores similares en muestras superficiales provenientes del bosque de Nothofagus (Musotto et al., 2012). Asimismo, se observa un aumento en la tasa de sedimentación y en el crecimiento de la turbera. Por otro lado, la disminución de Empetrum rubrum y Poaceae y el registro de Caltha, Astelia pumila, Donatia fascicularis y Myrteola nummularia sugieren el desarrollo de comunidades análogas con la Tundra Magallánica bajo condiciones paleoambientales muy frías y húmedas.

\subsection{Evolución paleoambiental del extremo este del canal Beagle}

\subsubsection{Tardiglacial tardío-Holoceno temprano}

Hacia los 12.000 años cal AP la configuración paleogeográfica en el sector de Punta Moat indicaría una mayor influencia marina por la probable presencia de la extensa bahía que se habría desarrollado al este de Punta Moat y el posible desarrollo de un paleolago hacia el oeste según muestra el modelo paleogeográfico (Fig. 7a). En Punta Moat, el ascenso relativo del nivel del mar y el consecuente ascenso del nivel de base, sumado a una probable disminución del caudal de los cursos de agua que recorrían la planicie glacifluvial por desaparición del hielo en los circos de las sierras Lucio López, habrían dado lugar a un drenaje deficiente con frecuentes encharcamientos. Esta situación y la escasa pendiente superficial de la planicie, probablemente habría promovido la formación de turberas en el área del valle inferior del río Moat. El registro polínico de la turbera Moat revela que las condiciones paleoecológicas de la misma, en sus estadios iniciales (con posterioridad a 11.200 años cal AP) habrían sido de minerotrofía, es decir, rica en nutrientes provenientes de sedimentos transportados por escurrimiento superficial, cursos de agua e inundaciones, favoreciendo el desarrollo de ciperáceas. La vegetación del área habría estado dominada por comunidades de gramíneas, hierbas, arbustos (Asteraceae subf. Asteroideae) y murtillares de Empetrum rubrum con escasos árboles de Nothofagus localizados regionalmente y alejados del sitio estudiado. Mientras tanto, hacia el oeste y sobre las tierras bajas a lo largo del canal Beagle, durante el Holoceno temprano, se expandían comunidades vegetales del ecotono bosque-estepa bajo condiciones ambientales más secas y cálidas que las actuales (Heusser, 1998; Markgraf y Huber, 2010). Hacia el este de Punta Moat, en el sector norte y sudoeste de la isla de los Estados, prevalecía aún la vegetación arbustiva y graminosa sin presencia de árboles (Ponce et al., 2011a; Björck et al., 2012).

Una vez que el agua de mar ingresó en el paleolago Picton, según nuestro modelo paleogeográfico hacia los 11.000 años cal AP, el paleolago se habría convertido en un fiordo que se extendía hacia el oeste, hasta las actuales islas Snipe y Becasses (Figs. 1b y 7b). De esta manera, la costa de Punta Moat se habría encontrado bajo una mayor influencia de aire marino. Hacia el sur permanecía aún emergida una porción 
de tierra, que limitaba la influencia directa de las corrientes de mar frías del sur sobre las costas del sector oriental del canal Beagle. El diagrama polínico de Moat 2 registra el paulatino incremento de Nothofagus a partir de los 10.500 años cal AP, que conforma comunidades ecotonales como respuesta a condiciones ambientales relativamente más húmedas, asociadas a una mayor disponibilidad de humedad efectiva, probablemente debido a la influencia local del spray marino. Un comportamiento similar se observa a partir de los 10.300 años cal AP en el suroeste de isla de los Estados (Fig. 1a), donde el registro del polen de Misodendrum da cuenta del desarrollo local del bosque de Nothofagus favorecido por un aumento en la humedad efectiva, acompañado de vegetación arbustiva y en cojín sobre la superficie de la turbera (Ponce et al., 2011a), mientras que en el norte de la isla de los Estados, el bosque de Nothofagus adquiere predominancia con posterioridad a los 9.850 años cal AP (Björck et al., 2012).

En Punta Moat se observa el desarrollo de un bosque denso de Nothofagus a partir de los 9.500 años cal AP, mientras que las condiciones minerotróficas de la turbera cambian y adquieren características ombrotróficas. La disminución de Cyperaceae y presencia de Sphagnum sugieren un incremento en la humedad efectiva y una disminución en el aporte de detritos minerales. Actualmente, las turberas ombrotróficas de Sphagnum se encuentran ubicadas en Tierra del Fuego entre las isohietas 450-600 mm de precipitación anual (Roig, 2004). Asimismo, el registro polínico de Astelia pumila, Drapetes muscosus y Donatia fascicularis, componentes típicos de las turberas en carpeta (Tundra Magallánica), podría ser el resultado del efecto de la brisa marina sobre la composición florística de la turbera. Actualmente, en las áreas costeras del sur de Chile $\left(52^{\circ} \mathrm{S}\right)$ se observa el desarrollo de turberas mixtas transicionales de Sphagnum y plantas en cojín debido a la influencia directa del spray marino, que aporta nutrientes minerales a la superficie de la turbera (Kleinebecker et al., 2008). La influencia directa de los vientos provenientes del mar desde el sur y sudoeste en el área debido a la desaparición de la bahía ubicada frente a las costas de Punta Moat, con la formación de los canales marinos Oglander y Richmond (Fig. 7c) y la consecuente formación de las islas Picton y Nueva hacia los 10.000 años cal AP según muestra nuestro modelo paleogeográfico (Fig. 7c), habría favorecido la llegada del spray marino con el aporte de nutrientes minerales a la turbera. El polen de Astelia pumila y Donatia fascicularis también es registrado desde sus inicios, a partir de los ca. 8.000 años cal AP (Heusser, 1995).

Hacia los 9.000 años cal AP, con la línea de costa ubicada a unos $2 \mathrm{~km}$ del sitio estudiado (Fig. 7d), las comunidades vegetales mantuvieron la fisonomía del bosque denso de Nothofagus y de turberas mixtas de transición con arbustos de Empetrum rubrum, colonizando las áreas más secas en los 'hummocks' de Sphagnum y vegetación palustre (Donatia fascicularis, Astelia pumila, Myrteola nummularia, Caltha y Tetroncium magellanicum) y graminoidea que sugieren niveles elevados de humedad en la superficie de la turbera.

\subsubsection{Holoceno medio}

El modelo paleogeográfico muestra que hacia los 7.000 años cal AP, el sector oriental del canal Beagle adquirió las características geográficas actuales. Hasta el momento, los depósitos marinos más antiguos hallados en el sector este de canal Beagle se localizan en la desembocadura del río Varela (Fig. 1b) con una edad basal de 7.100 años cal AP (Grill et al., 2002). Este fechado es coincidente con la configuración geográfica propuesta en nuestro modelo paleogeográfico para el sector este del canal Beagle.

Con posterioridad a los 6.800 años cal AP, las condiciones húmedas persistirían en el área de Punta Moat, intensificándose luego de los 6.000 años cal AP, y observándose un cambio en el paisaje con una mayor presencia del bosque denso de Nothofagus, como lo sugiere el aumento en los valores del influjo polínico, y el establecimiento de la tundra hiperhúmeda magallánica con desarrollo de turberas en carpeta con áreas inundadas sobre la superficie de la turbera. Estas condiciones de mayor humedad también son registradas, entre los $c a .7 .000$ y 6.500 años cal AP, en las localidades ubicadas al oeste de Punta Moat y a lo largo del canal Beagle (Heusser, 1998; Markgraf y Huber, 2010), con la expansión regional del bosque denso de Nothofagus como característica ambiental de importancia para la región. En el sudoeste de la isla de los Estados se observa un comportamiento similar con el paulatino desarrollo del Bosque Magallánico Siempreverde de Nothofagus y de la Tundra Magallánica a partir de los $c a$. 6.700 años cal AP bajo condiciones muy húmedas y frías (Ponce et al., 2011a). 


\section{Conclusiones}

Según el modelo paleogeográfico propuesto, una configuración similar a la actual del área de estudio se habría alcanzado hacia los 9.000 años cal AP, una vez que el mar inundara el paleolago Picton (11.000 años cal AP), lo que habría dado lugar al paleofiordo islas Snipe-Becasses, y causado la separación definitiva de las actuales islas Picton, Nueva y Lennox (10.000 años cal AP).

Sobre la base de las evidencias palinológicas y paleogeográficas analizadas se sugiere que la evolución paleoambiental y paleoclimática del extremo este del canal Beagle coincide, en líneas generales, con el modelo regional de instalación posglacial de comunidades vegetales en tierras desenglazadas en el sur de Tierra del Fuego, pero con ciertas características locales propias.

Este modelo regional de vegetación posglacial plantea la sucesión de comunidades pioneras graminosas y arbustivas propias de la estepa, seguidas del ecotono bosque-estepa y del bosque de Nothofagus (Heusser, 1998; Markgraf y Huber, 2010; Ponce et al., 2011a). Con el inicio de la deglaciación en Punta Moat y con el retiro de los paleoglaciares alpinos hacia las cabeceras de las sierras de Lucio López, se formó una planicie aluvial con cursos de agua que fluían en dirección perpendicular al actual canal sobre la que comenzó el desarrollo de la turbera muestreada. Hacia el oeste, en el sector central y occidental del canal Beagle, se desarrollaba mientras tanto una vegetación de estepa con escasos árboles de Nothofagus localizados regionalmente, bajo condiciones de menor humedad que la actual y paulatino ascenso de la temperatura. La evolución de la vegetación durante el Holoceno temprano en Punta Moat es similar a la que se desarrolló en los sectores occidental y central del canal Beagle, donde las primeras comunidades vegetales que cubrieron el paisaje consistían en gramíneas, hierbas y arbustos con una turbera de ciperáceas, seguidas por una vegetación análoga al ecotono bosque-estepa. Sin embargo, la influencia del ascenso relativo del nivel del mar y el consecuente aporte del spray marino favoreció el desarrollo temprano del bosque de Nothofagus en Punta Moat, y a los 9.500 años cal AP, el paisaje ya presentaba las características de un bosque denso de Nothofagus al tiempo que la turbera cambiaba a condiciones de ombrotrofía con presencia de Sphagnum y disminución de
Cyperacea. Asimismo, el registro polínico de taxa propios de la Tundra Magallánica (Astelia pumila, Donatia fascicularis, Drapetes muscosus) coincide con la apertura de los canales marinos Richmond y Oglander y la consecuente formación de las islas Picton y Nueva. Esta situación habría favorecido el ingreso directo del spray marino proporcionando los nutrientes minerales necesarios para la aparición de elementos florísticos típicos de las turberas en carpeta de Donatia-Astelia. En tanto, hacia el oeste de Punta Moat, en el área central del canal Beagle, aún prevalecían las comunidades vegetales del ecotono bosque-estepa. A partir de los 7.000 años cal AP el sector oriental del canal Beagle adquirió las características geográficas actuales al tiempo que el bosque denso de Nothofagus se extendía a lo largo del canal como evidencia de un incremento en la humedad efectiva. En Punta Moat y en el suroeste de la isla de los Estados, en coincidencia con el establecimiento de las condiciones paleogeográficas similares a las actuales, se desarrollaron las comunidades vegetales de la Tundra Magallánica y del Bosque Magallánico Siempreverde de Nothofagus, bajo condiciones muy húmedas y frías.

\section{Agradecimientos}

Los autores agradecen al Dr. F. Zangrando (CONICETCADIC) por su apoyo y colaboración en las tareas de campo y a la Dra. L.L. Musotto por su colaboración en la construcción de la curva de edad versus profundidad. Este manuscrito se realizó con el apoyo de la Agencia Nacional de Promoción Científica y Tecnológica (PICT Redes 67/02 y PICT 2280) y del National Polar Research Institute (Antarctica Dome-Fuji Ice-core Drilling). También se agradecen los comentarios realizados por el Dr. R. Kilian y por el evaluador anónimo, los que ayudaron a mejorar la calidad de este manuscrito.

\section{Referencias}

Birks, H.J.; Gordon, A.D. 1985. Numerical methods in Quaternary pollen analysis. Academic Press Inc. Ltd.: 313 p. London.

Björck, S.; Rundgren, M.; Ljung, K.; Unkel, I.; Wallin, Ä. 2012. Multi-proxy analyses of a peat bog on Isla de los Estados, easternmost Tierra delFuego: a unique record of the variable Southern Hemisphere Westerlies since the last deglaciation. Quaternary Science Reviews 42: 1-14. 
Bujalesky, G. 1998. Holocene coastal evolution of Tierra del Fuego, Argentina. Quaternaryof South America and Antarctic Peninsula 11: 247-282.

Bujalesky, G. 2007. Coastal geomorphology and evolution of Tierra del Fuego (Southern Argentina). Geologica Acta 5 (4): 337-362.

Bujalesky, G. 2011 The flood of the Beagle Valley (11,000 yr B.P.) Tierra del Fuego. Anales Instituto Patagonia 39 (1): 5-21.

Coronato, A. 1995. The last Pleistocene Glaciation in tributary valleys of the Beagle Channel, Southernmost South America. Quaternary of South America and Antarctic Peninsula 9: 173-182.

Coronato, A.; Meglioli, A.; Rabassa, J. 2004. Glaciations in the Magellan Straits and Tierra del Fuego, Southernmost South America. In Quaternary Glaciations-Extent and Chronology (Ehlers, J.; Gibbard, P.; editors). Quaternary Book Series, Part III: 45-48. Elsevier Publishers.

Faegri, K.; Iversen, J. 1989. Textbook of Pollen Analysis, $4^{\text {th }}$ edition. John Willey and Sons: 328 p. Copenhague.

Fleming, K.; Johnston, P.; Zwartz, D.; Yokoyama, Y.; Lambeck, K.; Chappell, J. 1998. Refining the eustatic sea-level curve since the Last Glacial Maximum using far- and intermediate-field sites. Earth and Planetary Science Letters 163: 327-342.

Fritz, C.; van Dijk, G.; Smolders, A.J.P.; Pancotto, V.A.; Elzenga, T.J.T.M.; Roelofs, J.G.M.; Grootjans, A.P. 2012. Nutrient additions in pristine Patagonian Sphagnum bog vegetation: can phosphorus addition alleviate the effects of increased nitrogen loads. Plant Biology 14: 491-499.

Gordillo, S.; Bujalesky, G.; Pirazzoli, P.; Rabassa, J; Saliège, J. 1992. Holocene raised beaches along the northern coast of the Beagle Channel, Tierra del Fuego, Argentina. Palaeogeography, Palaeoclimatology, Palaeoecology 99: 41-54.

Gordillo, S.; Coronato, A.; Rabassa, J. 1993. Late Quaternary evolution of a subantarctic paleofjord, Tierra del Fuego. Quaternary Science Reviews 12: 889-897.

Grill, S.; Borromei, A.M.; Quattrocchio, M.; Coronato, A.; Bujalesky, G.; Rabassa, J. 2002. Palynological and sedimentological analysis of Recent sediments from Río Varela, Beagle Channel, Tierra del Fuego, Argentina. Revista Española de Micropaleontología 34 (2): 145-161.

Grimm, E. 2004. TG View 2.0.2 Illinois State Museum. Research and Collection Center. Springfield, Illinois.

Heusser, C.J. 1989a. Climate and chronolgy of Antarctica and adjacent South America over the past 30,000 yr.
Palaeogeography, Palaeoclimatology, Palaeoecology 76: 31-37.

Heusser, C.J. 1989b. Late Quaternary Vegetation and Climate of Southern Tierra del Fuego. Quaternary Research 31: 396-406.

Heusser, C.J. 1990. Late-glacial and Holocene vegetation and climate of subantarctic South America. Review of Palaeobotany and Palynology 65: 9-15.

Heusser, C.J. 1995. Palaeoecology of a Donatia-Astelia cushion bog, Magellanic Moorland-Subantarctic Evergreen Forest transition, southern Tierra del Fuego, Argentina. Review of Palaeobotany and Palynology 89: 429-440.

Heusser, C.J. 1998. Deglacial paleoclimate of the American sector of the Southern Ocean: Late Glacial-Holocene records from the latitude of Canal Beagle $\left(55^{\circ} \mathrm{S}\right)$, Argentine Tierra del Fuego. Palaeogeography, Palaeoclimatology, Palaeoecology 141: 277-301.

Isla, F.I.; Bujalesky, G. 2008. Coastal Geology and morphology of Patagonia and Fueguian Archipielago. In The Late Cenozoic of Patagonia and Tierra del Fuego. (Rabassa, J.R.; editor). Elsevier Science Publishers 10: 227-240.

Kilian, R.; Lamy, F. 2012. A review of Glacial and Holocene paleoclimate records from southernmost Patagonia (49-55으. Quaternary Science Reviews 53: 1-23.

Kleinebecker, T.; Hölzel, N.; Vogel, A. 2007. Gradients of continentality and moisture in South Patagonian ombrotrophic peatland vegetation. Folia Geobotanica 42 : 363-382.

Kleinebecker, T.; Hölzel, N.; Vogel, A. 2008. South Patagonian ombrotrophic bog vegetation reflects biogeochemical gradients at the landscape level. Journal of vegetation science 19: 151-160.

Markgraf, V.; Huber, U.M. 2010. Late and postglacial vegetation and fire history in Southern Patagonia and Tierra del Fuego. Palaeogeography, Palaeoclimatology, Palaeoecology 293 (2): 351-366.

McCormac, F.G.; Hogg, A.G.; Blackwell, P.G.; Buck, C.E.; Higham, T.F.G.; Reimer, P.J. 2004. SHCal04 Southern Hemisphere Calibration 0-1000 cal BP. Radiocarbon 46: 1087-1092.

Moore, D. 1983. Flora of Tierra del Fuego. Nelson: 396 p. Oswestry.

Mörner, N.A. 1987. Sea level changes and tectonics in Tierra del Fuego. Bulletin of the International Union for Quaternary Research, Neotectonics Commission 10: p. 31. Stockholm.

Mörner, N.A. 1991. Holocene sea level changes in the Tierra del Fuego region. Sao Paulo, Boletin Instituto 
de Geociências da Universidade de São Paulo, Special Publication 8: 133-151.

Musotto, L.L.; Bianchinotti, M.V.; Borromei, A.M. 2012. Pollen and fungal remains as environmental indicators in surface sediments of Isla Grande de Tierra del Fuego, southernmost Patagonia. Palynology 36 (2): 162-179.

Overpeck, J.T.; Webb, T.; Prentice, I.C. 1985. Quantitative interpretation of fossil pollen spectra: dissimilary coefficients and methods of modern analogs. Quaternary Research 23: 87-108.

Pendall, E.; Markgraf, V.; White, J.W.C.; Dreier, M.; Kenny, R. 2001. Multiproxy record of late PleistoceneHolocene climate and vegetation change in Patagonia. Quaternary Research 55: 168-178.

Pisano, V.E. 1977. Fitogeografía de Fuego-Patagonia chilena. In Comunidades vegetales entre las latitudes 52 y $56^{\circ} \mathrm{S}$. Anales del Instituto de la Patagonia 8: 121-250. Punta Arenas.

Ponce, J.F. 2009. Palinología y geomorfología del Cenozoico tardío de la Isla de los Estados. Tesis Doctoral (Inédito), Universidad Nacional del Sur, Departamento de Geología, Bahía Blanca: 191 p. Argentina.

Ponce, J.F.; Borromei, A.M.; Rabassa, J.O.; Martínez, O. 2011a. Late Quaternary palaeoenvironmental change in western Staaten Island $\left(54.5^{\circ} \mathrm{S}, 64^{\circ} \mathrm{W}\right)$, Fuegian Archipelago. Quaternary International 233: 89-100.

Prentice, I.C. 1985. Pollen representation, source area, and basin size: toward a unified theory of pollen analysis. Quaternary Research 23: 76-86.

Prentice, I.C. 1986. Multivariate methods of data analysis. In Handbook of Holocene (Berglund, B.E.; editor). Palaeocology and Palaeohidrology. Wiley: 799-816. Chichester.

Rabassa, J. 1987. Lago Roca, Tierra del Fuego: The highest Holocene marine beach in Argentina? International Geoscience Programme Project 200, 'Late Quaternary sea-level correlation and applications'. Abstracts: p. 21. Ushuaia.

Rabassa, J. 2008. Late Cenozoic glaciations in Patagonia and Tierra del Fuego. In Late Cenozoic of Patagonia and Tierra del Fuego (Rabassa, J.; editor). Developments in Quaternary Sciences, Elsevier 11: 151-204. Amsterdam.

Rabassa, J.; Heusser, C.; Stuckenrath, R. 1986. New data on Holocene sea transgression in the Beagle Channel: Tierra del Fuego, Argentina. Quaternary of South America and Antarctic Península 4: 291-309.
Rabassa, J.; Serrat, M.; Martí, C.; Coronato, A. 1990. El Tardiglacial en el Canal Beagle, Tierra del Fuego, Argentina y Chile. In Congreso Geológico Argentino No. 11, Actas 1: 290-293. San Juan.

Rabassa, J.; Coronato, A.; Bujalesky, G.; Salemme, M.; Roig, C.; Meglioli, A.; Heusser, J.; Gordillo, S.; Roig, F.; Borromei, A.; Quattrocchio, M. 2000. Quaternary of Tierra del Fuego, Southernmost South America: an updated review. Quaternary International 68-71: 217-240.

Rabassa, J.; Coronato, A.; Roig, C.; Martínez, O.; Serrat, D. 2004. Un bosque sumergido en Bahía Sloggett, Tierra del Fuego, Argentina: evidencias de comportamiento tectónico diferencial en el Holoceno tardío. Procesos geomorfológicos y evolución costera. In Reunión de Geomorfología Litoral No. 2 (BlancoChao, R.; López Bedoya, J.; Pérez-Alberti, A.; editores.) Universidad de Santiago de Compostela, Actas: 333-346. Santiago de Compostela.

Roig, C.; Collado, L. 2004. Moat, provincia de Tierra del Fuego. In Los turbales de la Patagonia (Blanco, D.; de la Balze, V.; editores). Bases para su inventario y la conservación de su biodiversidad. Wetlands International 19: 66-69. Buenos Aires.

Stockmarr, J. 1971. Tablets with spores used in absolute pollen analysis. Pollen et Spores 13: 615-621.

Stuiver, M.; Reimer, P.J.; Reimer, R.W. 2005. Calib 5.0.2. http://calib.qub.ac.uk/calib/ (última visita 28-02-2014).

Trivi de Mandri, M.E.; Burry, L.S.; D’Antoni, H.L. 2006. Dispersión-depositación del polen actual en Tierra del Fuego, Argentina. Revista Mexicana de Biodiversidad 77: 89-95.

Tuhkanen, S. 1992. The climate of Tierra del Fuego from a vegetation geographical point of view and its ecoclimatic counterparts elsewhere. Acta Botánica Fennica 125: 4-17.

Tuhkanen, S.; Kuokka, I.; Hyvönen, J.; Stenroos, S.; Niemelä, J. 1989-1990. Tierra del Fuego as a target for biogeographical research in the past and present. Anales Instituto de la Patagonia 19: 1-107.

Zangrando, F.A.; Tessone, A.; Vázquez, M. 2009. El uso de espacios marginales en el archipiélago fueguino: implicancias de la evidencia arqueológica de Bahía Valentín. In Arqueología de la Patagonia, una mirada desde el último confín (Salemme, M.; Santiago, F.; Álvarez, M.; Piana, E.; Vázquez, M.; Mansur, M.E.; editores). Editorial Utopías: 47-62 p. Ushuaia. 